\title{
Results of Nephrectomy in Hypertension Associated with Unilateral Renal Disease
}

\author{
R. G. LUKE,* M.B., M.R.C.P., M.R.C.P.ED. ; A. C. KENNEDY, $\dagger$ M.D., F.R.C.P.ED., F.R.C.P.GLASG. \\ J. D. BRIGGS, $\ddagger$ M.B., M.R.C.P., M.R.C.P.ED., M.R.C.P.GLASG. ; N. W. STRUTHERS,§ CH.M., F.R.C.S., F.R.C.S.ED. \\ W. BARR STIRLING,§ CH.M., F.R.C.S.ED., F.R.C.S.GLASG.
}

Cummary : Nephrectomy has been carried out in 34 patients with hypertension associated with unilateral parenchymal renal disease (28 with unilateral pyelonephritis, 3 tuberculosis, 2 hypoplasia, and 1 adenocarcinoma). In 13 of the patients the blood pressure was corrected, in four it was improved, and in 17 it was unaffected. The intravenous pyelogram (by the infusion technique with nephrotomography if necessary) and renogram give adequate information in most patients with unilateral parenchymal renal disease but may need to be supplemerted by aortography, or retrograde pyelography, or divided renal function studies in a few special circumstances. When the function of the damaged kidney is less than $25 \%$ of the total (which is well maintained), and the contralateral kidney is intact, nephrectomy is recommended provided the hypertension is significant; success is more likely in younger patients with a short history of hypertension.

\section{Introduction}

We have previously reported (Luke et al., 1968) the results of surgical treatment in 27 patients with hypertension associated with renal artery stenosis; we now report the results of nephrectomy in 34 patients in whom hypertension was associated with unilateral parenchymal renal disease, mainly pyelonephritis. These are all of the patients selected for surgical management from a total of 102 with unilateral parenchymal renal disease. All the patients with presumed renovascular hypertension, whether due to renal artery stenosis or to unilateral parenchymal renal disease, were detected by screening about 1,000 patients with hypertension.

\section{Patients and Methods}

Our diagnostic policy has been outlined previously (Kennedy et al., 1965). Patients with significant hypertension were screened with an intravenous pyelogram and an isotope renogram for renal artery stenosis and for any form of unilateral parenchymal renal disease. Where the latter was suspected further procedures such as aortography, retrograde pyelography, and divided renal function studies were carried out provided surgical treatment seemed feasible. Recently infusion pyelography with tomography has proved very valuable in assessment and has lessened the need for more complex investigations.

Details of clinical features, results of investigation, and pathological findings in the 34 patients in whom nephrectomy was performed are shown in Table I. The last operation in the series was performed in January 1966. There were 24

\footnotetext{
* Lecturer in Medicine.

t Reader in Medicine.

$¥$ Registrar in Medicine.

Consultant Urologist.

Royal Infirmary, Glasgow C.4
}

females and 10 males, and the average age was 35 years. Mean preoperative diastolic blood pressure was $121 \mathrm{~mm}$. $\mathrm{Hg}$, with a range of 105 to $170 \mathrm{~mm}$. $\mathrm{Hg}$. Two patients (Cases 1 and 5) had grade 4 fundi and four (Cases 22, 27, 29, and 30) had grade 3 fundi. Eighteen patients showed E.C.G. changes suggestive of left ventricular hypertrophy or strain. In three patients (Cases 2, 4, and 24) hypertension was an incidental finding and in four it was impossible to estimate the duration of hypertension; in the remainder the mean time during which the blood pressure. had been known to be raised or relevant symptoms had been present was 18 months. Proteinuria was observed in 17 patients. Six (Cases 3, 4, 8, 14, 19, and 30) had significant bacteriuria (bacterial count $>10^{5}$ per $\mathrm{ml}$.) during the period of investigation; three of these six gave a past history of urinary tract infection. In a further seven patients there was also a history of urinary tract infection, often with loin pain. In 13 of the 17 patients who had been pregnant, hypertension either persisted after toxaemia of pregnancy or there was a history of recurrent toxaemia of pregnancy.

The intravenous pyelogram was abnormal on the affected side in every case, anci in 14 showed no evidence of function; renal calcification was seen in one patient with renal tuberculosis and in another with an adenocarcinoma. The radiological findings were regarded as consistent with pyelonephritis when there was caliceal clubbing and cortical scarring. In only one patient (Case 17) was there increased density on the affected side. The preoperative pyelogram appearances in the contralateral kidney were regarded as normal except in Case 3, where slight pyelonephritic changes were noted in the calices; but on follow-up, by which time infusion pyelography was being more commonly performed, slight caliceal or cortical changes were noted in three other patients (Cases 5, 8, and 26).

The isotope renogram was abnormal on the affected side in every patient except one (Case 32 ) in whom pyelonephritis was apparently confined to the lower pole of the kidney; she had a segmental nephrectomy. The remaining renogram patterns (see Chart) were of the A type (10 patients) or B type (12 patients), both indicating substantial impairment of function (Luke et al., 1966), or showed no function (10 patients). The renogram pattern was normal over the contralateral kidney in all patients except for three (Cases 11, 18, and 31) in whom there was slight delay in the third phase and in whom there was substantial impairment of total renal function.

Aortography showed gross abnormality of the parenchyma, with a patent non-stenosed renal artery, in all the 20 patients in whom it was performed, including Case 17. The opposite kidney was abnormal in two patients (Cases 3 and 10) in whom there was slight cortical scarring.

Divided renal function studies were carried out in nine patients (Cases 3, 4, 10,12,14,16,17, 18, and 29); usually a one- or two-hour collection was made, with intravenous urea infusion, for estimation of differential creatinine clearance and urinary sodium and urea concentration. Accurate 


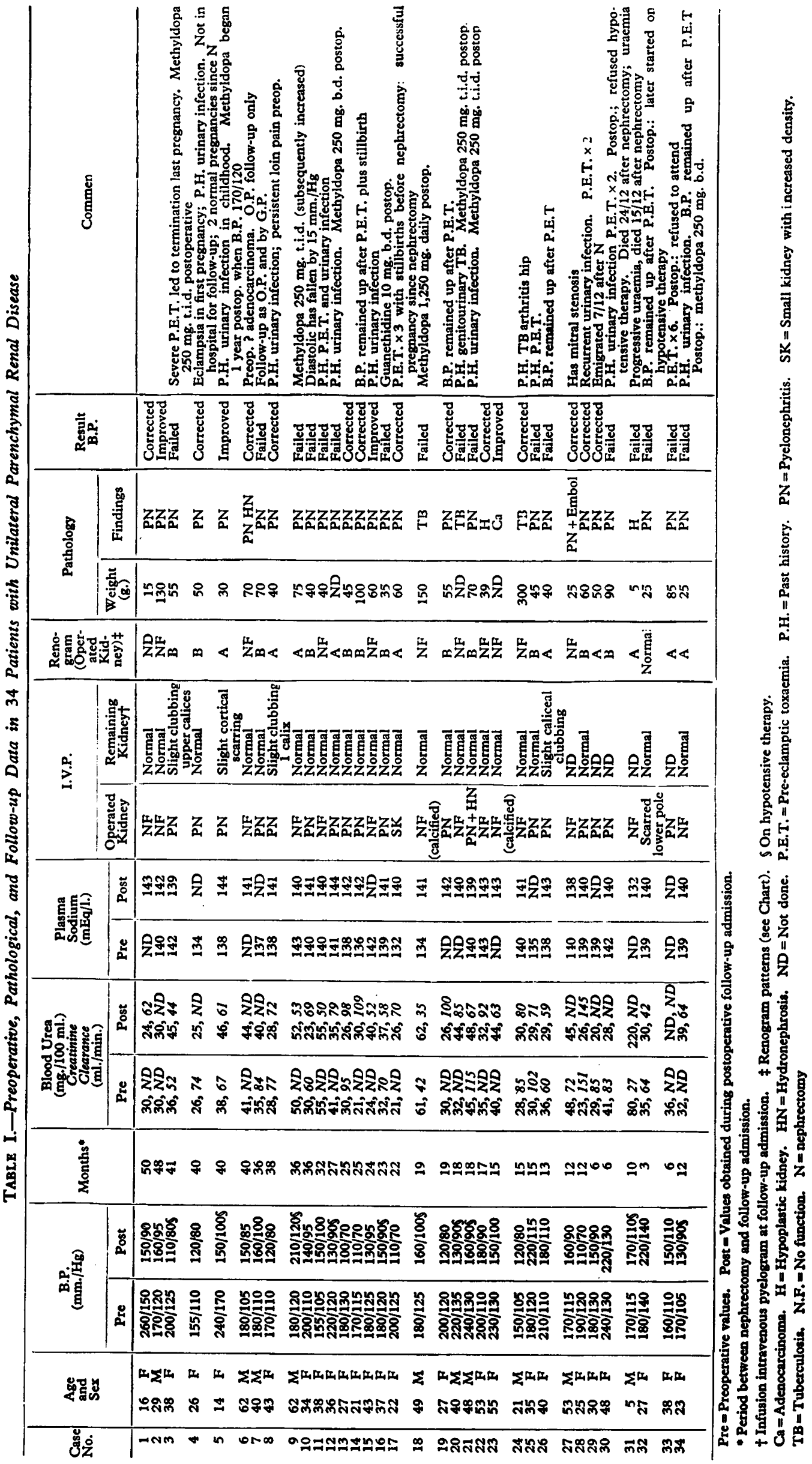


collections for clearance estimations were impossible in Case 12. In the others substantial impairment of function on the affected side was confirmed, the highest percentage of total function provided by the affected side being $27 \%$ (Case 14), with a mean of $19 \%$. In only one patient (Case 17) was the "ischaemic" pattern of result with a decreased urinary sodium and increased creatinine and urea concentration found; this was the only patient to show increased density in the intravenous pyelogram. Divided renal function tests were not usually carried out when the intravenous pyelogram and renogram suggested no function; but in eight of these patients retrograde pyelograms were performed for diagnostic purposes.

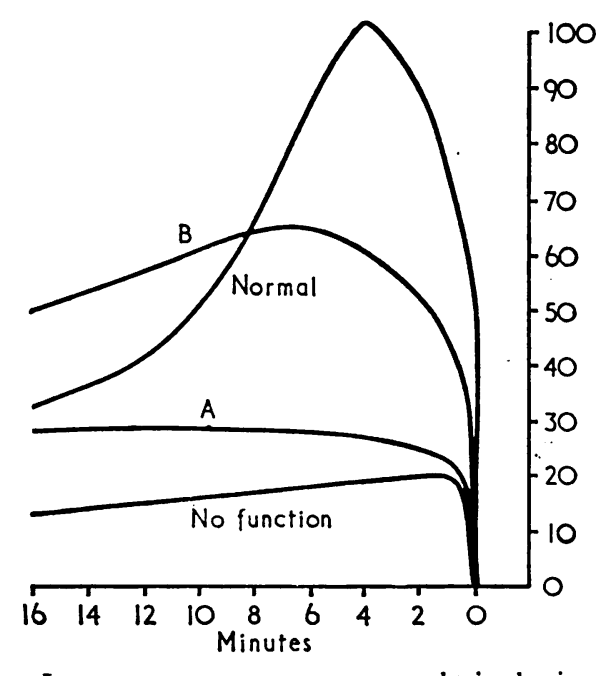

Isotope renogram patterns obtained in patients with unilateral parenchymal renal patients with unilateral parenchymal renal patients), no function (10 patients), normal (1 patient with lower pole pyelonephritis).

Mean preoperative blood urea was $36 \mathrm{mg} . / 100 \mathrm{ml}$., with a range of $21-80 \mathrm{mg} . / 100 \mathrm{ml}$.; in only three patients (Cases 11,18 , and 31) was the level above $50 \mathrm{mg} . / 100 \mathrm{ml}$. Mean preoperative endogenous creatinine clearance was $77 \mathrm{ml} . / \mathrm{min}$.

A decision to carry out nephrectomy was made only if the above investigations were considered to show that one kidney was very severely damaged, with either absent or severely impaired function, and that the other was normal or nearly so ; exceptions were Cases 11,18 , and 31 . Nephrectomy was carried out in 33 patients and a lower partial nephrectomy in one (Case 32).

The average weight of the removed kidney was $53 \mathrm{~g}$., with a range of 5-130 g., excluding two patients (Cases 18 and 24) with tuberculous kidneys weighing 150 and $300 \mathrm{~g}$. respectively. In 28 patients the histology was consistent with chronic pyelonephritis, and only one of these (Case 2) had a kidney weight of over $100 \mathrm{~g}$. In three patients (Cases 18, 20, and 24) tuberculosis was diagnosed on bacteriological and histological grounds, and they were given antituberculosis chemotherapy. In Case 23 an adenocarcinoma was confirmed by histology, and in Cases 22 and 31 a diagnosis of hypoplastic kidney was made because of the finding of small kidneys without specific histological changes.

Though in all cases the primary indication for investigation was hypertension, in six there were indications for nephrectomy in addition to the issue of renovascular hypertension. Three had unilateral tuberculosis with no function in the affected kidney, Case 23 had an adenocarcinoma, Case 6 was suspected of having an adenocarcinoma, and Case 8 had severe and persistent loin pain.

\section{Results}

Assessment of results was on the basis of regular outpatient attendances and a short admission at least one year after nephrectomy. The protocol on investigation during this admission was the same as that employed with patients who had renal artery stenosis (Luke et al., 1968) except that pyelography was carried out by means of the infusion technique in nearly all patients. It proved impossible to admit five of the 34 patients (Cases 2, 4, 7, 29, and 33). Follow-up data are given in Table I. The duration of follow-up was taken as the time interval between operation and the follow-up admission, whenever it was available. The mean time of follow-up was 27 months in the 17 patients in whom the blood pressure was corrected (13) or improved (4); in only one of these (Case 29), who emigrated, was the follow-up period less than 12 months. The mean time of follow-up in the 16 patients in whom operation had no effect on hypertension was 20 months; in four of these the period was less than 12 months. In assessing the effects of surgery on the blood pressure three terms are employed (Luke et al., 1968): (1) corrected-diastolic blood pressure of $90 \mathrm{~mm}$. $\mathrm{Hg}$ or less ; (2) improvement-fall in diastolic blood pressure of $20 \mathrm{~mm}$. $\mathrm{Hg}$ or more without hypotensive therapy; and (3) failure-others.

The results of surgery in the 34 operated cases are summarized in Table II. Hypertension was corrected or improved in $17(50 \%)$ of the 34 patients. There was no operative mortality or serious postoperative morbidity. Two patients (Cases 30 and 31) died with persistent hypertension and uraemia 24 and 15 months respectively after nephrectomy; one of these (Case 30) had refused to accept hypotensive therapy. Blood pressure was well controlled in six (Cases 3, $12,16,20,21$, and 34 ) by small doses of methyldopa or guanethidine and moderately well controlled in three (Cases 9, 18 , and 32) on various hypotensive drug regimens. One patient (Case 33) refused to attend for follow-up. Five patients (Cases $7,10,11,25$, and 26 ) were receiving no hypotensive therapy; in one of these (Case 10) there was a sustained fall of $15 \mathrm{~mm} . \mathrm{Hg}$ in the diastolic blood pressure after surgery. In the eight patients in the improved/corrected group who had preoperative hypertensive E.C.G. changes the E.C.G. became negative or the appearances became less marked.

TABLB II.-Effects of Nephrectomy* on Blood Pressure in 34 Patients With Unilateral Parenchymal Renal Disease



Partial nephrectomy in one patient. blood pressure of only $15 \mathrm{~mm} \mathrm{Hg}$.

In only two patients (Cases 8 and 14) was the urine culture positive on follow-up, and in both of these the cultures had also been positive before operation. None of the seven patients in whom proteinuria was detected before operation in the improved or corrected groups had proteinuria persisting on follow-up, as compared with 9 out of 10 with persistent proteinuria in the failed group. The renogram was normal in all patients in whom it was performed except for one (Case 31) who was uraemic (blood urea $220 \mathrm{mg} . / 100 \mathrm{ml}$.) Excluding Case 31 the mean follow-up blood urea was $36 \mathrm{mg} . / 100 \mathrm{ml}$. and the mean follow-up endogenous creatinine clearance was $67 \mathrm{ml} . / \mathrm{min}$. As in the previous description of the results of surgery in patients with renal artery stenosis a statistical comparison was made between the plasma sodium values preoperatively and those obtained on 
the routine follow-up admission in the patients having this measured on both occasions. The patients were split into two groups-those in whom the blood pressure had been corrected or improved, and those in whom there had been no effect on the blood pressure; there was a significant rise in the plasma sodium in the first group $(P<0.01)$ and no significant change in the second group $(\mathrm{P}>0.1)$.

\section{Discussion}

In a review of the literature Homer Smith (1956) found that hypertension was corrected by nephrectomy in only $26 \%$ of 575 patients with various types of unilateral parenchymal renal disease; but he stated that even this figure was probably an overestimate, since many unsuccessful cases had almost certainly not been reported. A rather more enthusiastic note was sounded in Britain by Yates-Bell (1959), who reported a personal series of 89 cases of nephrectomy for unilateral renal disease in hypertension; of the 66 cases that were acceptable for analysis approximately $50 \%$ had correction or improvement of hypertension. As over the past decade interest has grown in renal artery stenosis as a surgically remediable cause of hypertension, fewer reports of the results of surgical treatment of hypertension due to unilateral parenchymal renal disease have appeared (Gifford et al., 1965 ; Kaufman and Goodwin, 1965 ; Parsons et al., 1965; Redwood, 1967) and there is not at the present time agreement either on the frequency of unilateral parenchymal renal disease as a cause of hypertension or on the role of surgery.

' the combination of intravenous pyelogram and isotope renogram which we have used to discover cases of renal artery stenosis is equally effective in detecting cases of unilateral parenchymal renal disease. When this method was applied to a relatively unselected hypertensive population (Kennedy et al., 1965) we found more patients in whom hypertension was associated with unilateral parenchymal renal disease than with renal artery stenosis. A smaller proportion of these patients were selected for surgery (34 out of 102 for those with parenchymal renal disease compared with 27 out of 41 for those with renal artery stenosis) and the results of surgery are distinctly less good ( $50 \%$ correction-plus-improvement rate in parenchymal renal disease compared with $85 \%$ in renal artery stenosis). Nevertheless we cannot agree with Stamey (1965) when he says that "the relationship between [unilateral] pyelonephritis and hypertension is probably of little importance"; we believe that worth-while results for surgery can be achieved in patients with unilateral parenchymal renal disease but that proper selection of cases is important, especially if patients are not to lose functioning renal tissue unnecessarily.

\section{Selection of Cases}

The past history is more often helpful than in renal artery stenosis. Ten of the 34 patients gave a history of urinary tract infection; loin pain is worthy of note. As in the patients with renal artery stenosis, we were impressed with the frequency of a history of hypertension dating back to toxaemia of pregnancy or of a history of recurrent toxaemia of pregnancy. In one of the cases of renal tuberculosis there was a history of tuberculous arthritis in childhood, and in another a history of genitourinary tuberculosis.

Proteinuria was a more common finding in this group of patients than in those with renal artery stenosis (50\% as compared with $18 \%$ ).

It is important at the outset to decide whether the hypertension is significant enough to merit investigation, whether a surgical approach would be feasible, and whether total renal function is at an adequate level to permit nephrectomy and to indicate that the contralateral kidney has been spared by the pathological process affecting the damaged kidney. In general, consideration of a surgical approach in a patient with apparently unilateral renal parenchymal disease is not worth pursuing unless the blood urea is under $50 \mathrm{mg} . / 100 \mathrm{ml}$. and the creatinine clearance above $50-55 \mathrm{ml} . / \mathrm{min}$. In the present series nephrectomy was carried out in three patients with blood ureas over $50 \mathrm{mg} . / 100 \mathrm{ml}$.; a non-functioning kidney was removed on each occasion and no beneficial effect on the blood pressure was obtained. It is, however, sometimes difficult to deny a patient the possible, even though highly improbable, benefit from nephrectomy when the damaged kidney is contributing nothing to renal excretory function; this was so in Case 31, a boy of 5 years with substantial renal impairment from whom a hypoplastic kidney weighing $5 \mathrm{~g}$. was removed without benefit.

Few, if any, cases of unilateral parenchymal renal disease fail to be detected by the combined use of the intravenous pyelogram and the isotope renogram. Infusion pyelography, combined with renal tomography, is of value in defining the type of renal disease, and retrograde pyelography and/or aortography can now usually be reserved, firstly, for cases with a small kidney and a normal pelvicaliceal system, when renal artery stenosis may be present; secondly, where no renal tissue has otherwise been shown; and, thirdly, where doubt about the possibility of, for example, renal parenchymal neoplasm persists. While it is relatively easy to show that one kidney is damaged and functioning poorly, it is more difficult to prove that the contralateral organ is not affected by pyelonephritis. Helpful points are well-maintained total renal function, normal or increased renal size, smooth cortical outline, normal pelvicaliceal system, and a normal isotope renogram pattern.

Of the four patients (Cases 4, 14, 17, and 29) in whom hypertension was corrected by nephrectomy and in whom divided renal function tests were carried out, only one (Case 17) showed an "ischaemic" pattern of results with a reduction in urine volume and sodium concentration, and an increase in creatinine and urea concentration, on the affected side. The absence of the "ischaemic" pattern in divided renal function tests in a patient with a successful result from nephrectomy for pyelonephritis was also observed by Maxwell and Prozan (1962). Thus the only value of divided renal function tests is to quantitate the contribution of the damaged kidney to total renal function. As a result of our experience we feel that a reasonably adequate assessment of the contribution of the damaged kidney to total renal function can be made in the majority of cases by a combination of pyelography and the isotope renogram, though in some circumstances divided function studies may be required; we prefer to avoid the use of divided renal function tests wherever possible, however, in view of the potential hazard of introducing infection into the sound kidney.

\section{Comparison of Successes and Failures}

The hypertensive patients who benefited from nephrectomy were younger (mean age 33.3 compared with 37.3 years; this difference is not statistically significant), had a shorter history of hypertension (10 as compared with 28 months, $\mathrm{P}<0.05$ ), and had a lower blood urea before operation $(31 \mathrm{mg} . / 100 \mathrm{ml}$. as compared with $43 \mathrm{mg}$. $/ 100 \mathrm{ml}$., $\mathrm{P}<0.01$ ) than those in whom the operation was without effect on the blood pressure. Responses were obtained whether the removed kidney had been functioning or not, as assessed by the pyelogram and renogram, and were also obtained in a proportion of patients whether the pathological diagnosis was pyelonephritis, hypoplasia, adenocarcinoma, or tuberculosis. Kaufman and Good- 
win (1965) claimed that nephrectomy for tuberculosis was associated with a much better correction rate than was the case with pyelonephritis, but this was not observed in the present series, only one of the three patients from whom a tuberculous kidney was removed obtaining an effect on the blood pressure. In only one patient in this series was a partial nephrectomy performed-there was no effect on the blood pressure.

\section{Indications for Nephrectomy}

When the contralateral kidney appears normal, total renal function is good, and the hypertension is significant, removal of a non-functioning kidney is usually justified. In such patients there may be other indications for nephrectomy, as previously noted, in addition to the renovascular issue. Nephrectomy for a non-functioning pyelonephritic kidney has been held to be indicated even in a normotensive patient (Krivec, 1967) to prevent the possible spread of the disease to the other kidney.

A decision to carry out nephrectomy is much more difficult where some excretory function persists in the affected kidney. The mechanism of hypertension found in some patients with unilateral parenchymal renal disease is not yet understood, though the work of Kincaid-Smith (1955) has suggested that it may be secondary to intrarenal arterial narrowing. It is of interest that, as with the patients with renal artery stenosis, there was a significant rise in plasma sodium in those of this series in whom the blood pressure was influenced by nephrectomy, but not in the failed group. Since Brown et al. (1965) have shown a reciprocal relationship between the plasma sodium and the plasma renin, the renin-angiotensin mechanism may play a part in the hypertension associated with some cases of unilateral parenchymal renal disease. Nevertheless at present there is no certain way of detecting preoperatively which patients will respond to nephrectomy, and nephrectomy in these circumstances still remains "an experimental procedure" (Homer Smith, 1956).

We now believe that a functioning but diseased kidney should be removed in an effort to correct hypertension only when it is severely damaged and is contributing less than $25 \%$ to total renal function, which itself should be well maintained, and when there is no evidence of significant disease in the contralateral organ. Acceptance of this policy is possibly associated with the loss of some opportunities to correct hypertension because of the non-removal of less severely functionally impaired kidneys, but it is nevertheless, in our opinion, a reasonable policy at the present stage of our knowledge. If the affected kidney is contributing about $25 \%$ to overall renal function the patient may be followed over a period and serial observations made on blood pressure, if necessary, on hypotensive therapy, on renal function, and on the findings of the isotope renogram and intravenous pyelogram.

\section{Conclusions}

As a result of our experiences in the detection of cases of $\overrightarrow{\vec{F}}$ unilateral parenchymal renal disease and the selection of such patients for surgery, we suggest the following policy:

(1) Assess the significance of the hypertension, total renalow function, and fitness for possible surgery.

(2) In suitable cases proceed to intravenous pyelogram ando renogram.

(3) An infusion intravenous pyelogram with nephrotomo-. graphy will often permit more precise diagnosis and show $\vec{\omega}$ more clearly the anatomy of the contralateral kidney. Aorto-o graphy, retrograde pyelography, and divided renal function $\frac{3}{3}$ tests will only occasionally be necessary.

(4) If the affected kidney is contributing less than $25 \%$ to total renal function, which is itself well maintained, andN the contralateral kidney is intact, nephrectomy is advised, $\overrightarrow{-}$ provided the hypertension is significant. The best results are likely to be obtained in younger patients with a shorto history of hypertension.

Many of the patients in this series were referred to us by members of the hypertension unit, department of cardiology, by the 3 physicians in Glasgow Royal Infirmary, and by physicians in other hospitals in Glasgow and the West of Scotland ; we are grateful to them, and in particular to Dr. R. Fife, for their co-operation. $\vec{C}$ We would also like to thank Dr. W. P. Duguid, of the departmento of pathology, Royal Infirmary, and those radiologists who carried out some of the investigations before referral.

\section{REFERENCES} Brown, J. J., Davies, D. L., Lever, A. F., and Robertson, J. I. S. (1965).

Gifford, R. W., McCormack, L. J., and Poutasse, E. F. (1965). Proc Mayo Clin., 40, 834

Kaufman, J. J., and Goodwin, W. E. (1965). Amer. F. Med., 38, 337. Kennedy, A. C., Luke, R. G., Briggs, J. D., and Stirling, W. B. (1965)

Kincaid-Smith, P. (1955). Lancet, 2, 1263.

Krivec, O. (1967). 14 Kongres der Internationalen Gesellschaft für uro-s logie, p. 375. Paris.

Luke, R. G., Briggs, J. D., Kennedy, A. C., and Stirling, W. B. (1966) Quart. ऊ. Med., 35, 237.

Luke, R. G., et al.' (1968). Brit. med. ₹., 2, 76.

Maxwell, M. H., and Prozan, G. B. (1962). Progr. cardiovasc. Dis., $5 \hat{\text { 尺े }}$

Parsons, V., Cameron, J. S., and Gray, B. (1965). Guy's Hosp. Rev., 114, 114.

Redwood, D. R. (1967). Brit. F. Urol., 39, 153.

Smith, H. W. (1956). F. Urol. (Baltimore), 76, 685

Smith, H. W. (1956). F. Urol. (Baltimore), 76,

Yates-Bell, J. G. (1959). Brit. med. F., 2, 1371. 